DPSU-97-5

KEK Preprint 97-11

May, 1997

\title{
Probing $\mu$-term Generation Mechanism in String Models
}

\author{
Yoshiharu Kawamura ${ }^{a}$ [, Tatsuo Kobayashi ${ }^{b}$ \& \\ and \\ Manabu Watanabe ${ }^{a}$ p \\ ${ }^{a}$ Department of Physics, Shinshu University \\ Matsumoto 390, Japan \\ and \\ ${ }^{b}$ Institute of Particle and Nuclear Studies \\ High Energy Accelerator Research Organization \\ Midori-cho, Tanashi, Tokyo 188, Japan
}

\begin{abstract}
We give a generic method to select a realistic $\mu$-term generation mechanism based on the radiative electroweak symmetry breaking scenario and study which type is hopeful within the framework of string theory. We discuss effects of the moduli $F$-term condensation and $D$-term contribution to soft scalar masses.
\end{abstract}

\footnotetext{
${ }^{1}$ e-mail: ykawamu@gipac.shinshu-u.ac.jp

${ }^{2}$ e-mail: kobayast@tanashi.kek.jp

${ }^{3}$ e-mail: watanabe@azusa.shinshu-u.ac.jp
} 


\section{Introduction}

One of important problems in the supersymmetric standard model (SUSYSM) is how a SUSY Higgs mass term, the $\mu$-term, is derived naturally within the framework of supergravity (SUGRA). Because the $\mu$-term is not a soft SUSY breaking term and a natural order of such a mass $\mu$ is the gravitational scale $M$ in SUGRA This problem is called the $\mu$-problem [四. Some types of natural mechanisms of $\mu$-term generation have been proposed, e.g. the case with Kähler potential including a Higgs mixing term [2] and a superpotential including a suitably suppressed mass term [3, 1, 4, 5].

In addition to the supersymmetric mass term, soft SUSY breaking mass terms also contribute to the Higgs mass terms. Explication of SUSY breaking mechanism is an important and unsolved problem. Superstring theory (SST) is only the known candidate for unified theory including gravity and is expected to give a natural solution due to some non-perturbative effects. Recently there have been various remarkable developments in studying nonperturbative aspects of SST [6], but SUSY breaking mechanism has not been fully understood yet. For the present, we can derive formulae for soft SUSY breaking terms assuming the existence of nonperturbative superpotential which induces SUSY breaking in string models [7, 8]. Further these soft terms can be parametrized simply using several parameters in most cases. For example, under the assumption that only the dilaton field $S$ and/or the overall moduli field $T$ trigger SUSY breaking by their $F$-term condensations, soft scalar masses, gaugino masses and $A$-parameters are written model-independently by the gravitino mass $m_{3 / 2}$ and the goldstino angle $\theta$ in the case with the vanishing vacuum energy [9]. We can extend such a parametrization into cases with several moduli fields [10, 11]. On the other hand, the $B$-parameter depends on the $\mu$-term generation mechanisms $[8,9,12]$. Therefore $\mu$ and $B$ parameters are usually treated as arbitrary parameters. The more arbitrary parameters exist, the harder we analyze and the less we have a predictivity. It is important to select $\mu$-term generation mechanism in some way.

In this paper, we study several $\mu$-term generation mechanisms based on the radiative breaking scenario of electroweak symmetry breaking within the framework of string theory. Examining the parameter space leading to suc-

\footnotetext{
* Through this paper, we take $M=1$.
} 
cessful electroweak symmetry breaking, we probe a realistic $\mu$-term generation mechanism. We take into account $D$-term contributions to soft scalar masses [13] in our analysis.' The reason is as follows. String models, in general, have extra gauge symmetries including an anomalous $U(1)$ symmetry and extra massless matter fields other than the SUSY-SM ones. We can reduce gauge group and massless spectrum through symmetry breaking along flat directions [16]. As discussed in recent papers [17], sizable $D$-term contributions can appear through such flat direction breaking.

This paper is organized as follows. In the next section, we give our basic assumptions, formulae of $\mu$-term and $B$-term, and our strategy. In section 3 , we study several types of $\mu$-term generation mechanisms based on the radiative breaking scenario and show which type is hopeful. Section 4 is devoted to conclusions and discussions.

\section{Method of Analysis}

\subsection{Basic assumptions}

We take the SUSY-SM with soft SUSY breaking parameters expected to be derived as a low-energy theory from orbifold models through flat direction breaking as a starting point of our analysis. Let us first list our basic assumptions.

1. The SUSY is broken by the F-term condensations of dilaton field $S$ and/or moduli fields $T_{m}$.

2. The vacuum energy $V_{0}$ vanishes, i.e., $V_{0}=0$, at the tree level.

3. For the third family of the quark doublet $q_{3}$, the up-type singlet $t$ and the Higgs doublet $H_{2}$, their modular weights are $n_{k}=-1$.

4. The top Yukawa coupling $f_{t}$ does not depend on moduli fields, i.e., $\partial f_{t} / \partial T_{m}=0$.

5. The modular weight of the Higgs doublet $H_{1}$ is -1 (or -2 ).

${ }^{\dagger}$ Study on the Higgs sector has been done, e.g. within the framework of string models without $D$-term contribution 14] and SUSY grand unified theory (SUSY-GUT) with $D$ term contributions [15]. 
6. The product $H_{1} H_{2}$ of Higgs doublets is gauge invariant under all gauge symmetries which are broken along a flat direction.

7. Effects of moduli-dependent threshold corrections for gauge couplings and gaugino masses are negligibly small.

8. The Kac-Moody levels satisfy $k_{\alpha}=1$.

9. The gaugino masses $M_{\alpha}, B$ and $\mu$ are all real.

10. The dependence of dilaton and moduli fields is small in the $\mu$-parameter, i.e., $\partial \mu / \partial S, \partial \mu / \partial T_{m} \ll 1$.

The third and fourth assumptions can be justified from the fact that the coupling $f_{t}$ is strong and allowed as a renormalized coupling in the untwisted sector.

Here we discuss the overall-moduli case $\left(T=T_{1}=T_{2}=T_{3}\right)$ for simplicity. Under the above assumptions, we can obtain the following formulae of soft scalar masses $m_{k}^{(0) 2}$, the $A$-parameter $A_{t}^{(0)}$ among $\tilde{q}_{3}, \tilde{t}$ and $H_{2}$, and the gaugino mass $M_{1 / 2}^{(0)}$,

$$
\begin{aligned}
& m_{k}^{(0) 2}=m_{3 / 2}^{2}\left(1+n_{k} \cos ^{2} \theta\right)+d_{k} m_{3 / 2}^{2}, \\
& A_{t}^{(0)}=-\sqrt{3} m_{3 / 2} e^{-i \alpha_{S}} \sin \theta \\
& M_{1 / 2}^{(0)}=\sqrt{3} m_{3 / 2} e^{-i \alpha_{S}} \sin \theta
\end{aligned}
$$

where we use the following parameterization

$$
\begin{aligned}
& \left\langle\left(K_{S}^{S}\right)^{1 / 2} F^{S}\right\rangle=\sqrt{3} m_{3 / 2} e^{i \alpha_{S}} \sin \theta, \\
& \left\langle\left(K_{T}^{T}\right)^{1 / 2} F^{T}\right\rangle=m_{3 / 2} e^{i \alpha_{T}} \cos \theta .
\end{aligned}
$$

Here $d_{k} m_{3 / 2}^{2}$ is a $D$-term contribution and we get the relation $d_{H_{1}} m_{3 / 2}^{2}+$ $d_{H_{2}} m_{3 / 2}^{2}=0$ from the assumption 6 and the fact that its contribution is proportional to broken diagonal charges of scalar field. The natural order of $d_{k}$ is expected to be $O(1)$ from some explicit models [17].

In the limit of the moduli dominant SUSY breaking, we can not neglect moduli-dependent threshold corrections, but we do not consider such a case for simplicity in this paper. 
The top quark mass is given as $m_{t}\left(m_{t}\right)=f_{t}\left(m_{t}\right) v|\sin \beta| / \sqrt{2^{\ddagger}}$ where $\tan \beta=\left\langle h_{2}\right\rangle /\left\langle h_{1}\right\rangle$ and $v^{2}=\left\langle h_{2}\right\rangle^{2}+\left\langle h_{1}\right\rangle^{2}$. $\left(h_{i}\right.$ 's are neutral components of $H_{i}$ 's.) We use the value $m_{t}=175 \mathrm{GeV}$ as the top quark mass from the current experiments. Through our analysis, we take the gauge coupling unification scale $M_{X}=1.7 \times 10^{16} \mathrm{GeV}$ as an energy scale where boundary conditions are imposed. Hence it is supposed that the above formulae (11)-(3) hold at $M_{X}$.

By the use of renormalization group equations (RGEs) of the SUSY-SM [19, 20], soft scalar masses of $H_{1}$ and $H_{2}$ at the weak scale (we use the $Z$-boson mass $M_{Z}$ ) are given as,

$$
\begin{aligned}
& m_{H_{1}}^{2}=h m_{3 / 2}^{2}+d m_{3 / 2}^{2}, \\
& m_{H_{2}}^{2}=\bar{h} m_{3 / 2}^{2}-d m_{3 / 2}^{2}
\end{aligned}
$$

where

$$
\begin{aligned}
& h=2.56 \sin ^{2} \theta+\left(1+n_{H_{1}}\right) \cos ^{2} \theta, \\
& \bar{h}=2.56 \sin ^{2} \theta-3 I_{\Sigma} \sin ^{2} \theta
\end{aligned}
$$

and $d \equiv d_{H_{1}}=-d_{H_{2}}$. Here the second term in RHS of (9) represents the effect of the top Yukawa coupling and $I_{\Sigma}$ is a function of $\tan \beta$ (or the top Yukawa coupling). The values of $\alpha_{t}\left(\equiv f_{t}^{2} / 4 \pi\right)$ and $I_{\Sigma}$ are given in Table 1 .

\section{$2.2 \mu$-parameter and $B$-parameter}

Several types of solutions for the $\mu$-problem have been proposed [21]. Here we explain some of them briefly and we give formulae of the $B$-parameter for each $\mu$-term generation mechanism.

( $\mu$-1) The $\mu$-term $\mu_{Z}$ of $O\left(m_{3 / 2}\right)$ appears after SUSY breaking in the case where a Kähler potential includes a term such as $Z_{1} H_{2}$ [2]. In this case we have

$$
\mu_{Z}=m_{3 / 2}\langle Z\rangle-\left\langle F_{T}\right\rangle\left\langle Z^{T}\right\rangle
$$

$\ddagger$ The pole mass of top quark is related with the running mass as

$$
m_{t}^{\text {pole }}=m_{t}\left(m_{t}\right)\left[1+\frac{4 \alpha_{3}\left(m_{t}\right)}{3 \pi}+O\left(\alpha_{3}^{2}\right)\right] .
$$




$$
\begin{aligned}
B_{Z}= & 2 m_{3 / 2}+\left\langle F_{T}\right\rangle\left(\partial^{T} \log \mu_{Z}-\frac{n_{H_{1}}+n_{H_{2}}}{\left\langle T+T^{*}\right\rangle}\right) \\
& +\frac{m_{3 / 2}}{\mu_{Z}}\left\langle F_{T}\right\rangle\left\langle Z^{T}\right\rangle .
\end{aligned}
$$

Hereafter we take $Z=1 /\left(T+T^{*}\right)$ and then $\mu_{Z}$ and $B_{Z}$ are given as

$$
\begin{aligned}
\mu_{Z}= & m_{3 / 2}\left(1+e^{i \alpha_{T}} \cos \theta\right), \\
B_{Z}= & \frac{m_{3 / 2}}{1+e^{i \alpha_{T}} \cos \theta}\left\{2-\cos \theta\left(e^{-i \alpha_{T}}\left(1+n_{H_{1}}+n_{H_{2}}\right)-e^{i \alpha_{T}}\right)\right. \\
& \left.-\cos ^{2} \theta\left(2+n_{H_{1}}+n_{H_{2}}\right)\right\} .
\end{aligned}
$$

( $\mu$-2) The $\mu$-term $\mu_{\lambda}$ of $O\left(m_{3 / 2}\right)$ appears after SUSY breaking in the case where a superpotential $W$ includes a term such as $\lambda \tilde{W} H_{1} H_{2}$ [5]. Here $\tilde{W}$ is a superpotential which induces SUSY breaking. In this case we have

$$
\begin{aligned}
\mu_{\lambda} & =\lambda m_{3 / 2}, \\
B_{\lambda} & =m_{3 / 2}\left\{2-e^{-i \alpha_{T}} \cos \theta\left(n_{H_{1}}+n_{H_{2}}-\left\langle T+T^{*}\right\rangle\left\langle\partial^{T} \log \lambda\right\rangle\right)\right\} .
\end{aligned}
$$

( $\mu$-3) The $\mu$-term $\mu_{\mu}$ can be generated through some non-perturbative effects such as gaugino condensation [4] and it generally depends on the VEVs of $S$ and $T$. In this case we have

$$
\begin{aligned}
\mu_{\mu}= & \mu_{\mu}(S, T), \\
B_{\mu}= & m_{3 / 2}\left\{-1-\sqrt{3} e^{-i \alpha_{S}} \sin \theta\left(1-\left\langle S+S^{*}\right\rangle\left\langle\partial_{S} \log \mu_{\mu}\right\rangle\right)\right. \\
& \left.-e^{-i \alpha_{T}} \cos \theta\left(3+n_{H_{1}}+n_{H_{2}}-\left\langle T+T^{*}\right\rangle\left\langle\partial^{T} \log \mu_{\mu}\right\rangle\right)\right\} .
\end{aligned}
$$

$(\mu-4)$ In the model with a singlet field $N$ which has a coupling $f_{N} N H_{1} H_{2}$, the $\mu$-term appears when the field $N$ develops its VEV at some lower energy scale near $O\left(m_{3 / 2}\right)$ [3]. As a result, the $B$-parameter can be generated from the $A$-term $A_{N} f_{N} N H_{1} H_{2}$.

There can be an admixture of several $\mu$-term generation mechanisms and, in this case, $\mu$ and $B$ parameters are given as

$$
\begin{aligned}
\mu_{\mathrm{Mix}} & =\sum_{p} \mu_{p} \\
B_{\mathrm{Mix}} & =\sum_{p} \mu_{p} B_{p} / \sum_{q} \mu_{q}
\end{aligned}
$$


where the indices $p$ and $q$ run over all $\mu$-term generation mechanisms.

Finally we discuss a multi-moduli case briefly. $Z_{2 n}$ and $Z_{2 n} \times Z_{M}$ orbifold models [22] have $U$-type of moduli fields corresponding to complex structures of orbifolds 23 and a mixing term in the Kähler potential as

$$
\frac{1}{\left(T_{3}+T_{3}^{*}\right)\left(U_{3}+U_{3}^{*}\right)}\left(H_{1} H_{2}+\text { h.c. }\right) \text {. }
$$

In this case, the Higgs fields $H_{1}$ and $H_{2}$ belong to the untwisted sector. We assume $F$-terms of $S, T_{i}(i=1,2,3)$ and $U_{3}$ contribute SUSY breaking and these are parametrized by $m_{3 / 2}, \theta, \Theta_{3}$ and $\Theta_{3}^{\prime}$ following Refs. [9, 10, 11]. Then the soft scalar masses, the $\mu$-term and $B$-term are written at the tree level as 11, 14,

$$
\begin{aligned}
& m_{H_{1}}^{(0) 2}=m_{3 / 2}^{2}\left(1-3 \cos ^{2} \theta\left(\Theta_{3}^{2}+\Theta_{3}^{\prime 2}\right)\right)+d m_{3 / 2}^{2}, \\
& m_{H_{2}}^{(0) 2}=m_{3 / 2}^{2}\left(1-3 \cos ^{2} \theta\left(\Theta_{3}^{2}+\Theta_{3}^{\prime 2}\right)\right)-d m_{3 / 2}^{2}, \\
& \mu_{M}=m_{3 / 2}\left(1+\sqrt{3} \cos \theta\left(e^{i \alpha_{T_{3}} \Theta_{3}}+e^{i \alpha_{T_{3}^{\prime}} \Theta_{3}^{\prime}}\right)\right), \\
& \mu_{M} B_{M}=2 m_{3 / 2}^{2}\left(1+\sqrt{3} \cos \theta e^{i \alpha_{T_{3}}} \Theta_{3}\right)\left(1+\sqrt{3} \cos \theta e^{i \alpha_{T_{3}^{\prime}} \Theta_{3}^{\prime}}\right) .
\end{aligned}
$$

It is easy to show that $B_{M}$ and $\mu_{M}$ reduce to $B_{Z}$ and $\mu_{Z}$, respectively if we set $\Theta_{3}=1 / \sqrt{3}$ and $\Theta_{3}^{\prime}=0$.

We give formulae of $\mu / m_{3 / 2}$ and $B / m_{3 / 2}$ in Table 2 . Here we choose $e^{-i \alpha_{S}}=1, e^{-i \alpha_{T_{i}}}=e^{i \alpha_{T_{i}}}= \pm 1$ and $n_{H_{1}}=-1$ and use this choice in our analysis. In general, the $B / m_{3 / 2}$ contains a small number of free parameters compared with $\mu / m_{3 / 2}$ and so the analysis of $B / m_{3 / 2}$ can be more predictable.

\subsection{Strategy}

In this subsection, we give an outline of our strategy to probe $\mu$-term generation mechanism based on the radiative electroweak symmetry breaking scenario. The neutral fields $h_{1}$ and $h_{2}$ have the following potential [24]:

$$
\begin{aligned}
V\left(h_{1}, h_{2}\right) & =m_{1}^{2} h_{1}^{2}+m_{2}^{2} h_{2}^{2}+\left(\mu B h_{1} h_{2}+h . c .\right) \\
& +\frac{1}{8}\left(g^{2}+g^{2}\right)\left(h_{1}^{2}-h_{2}^{2}\right)^{2}, \\
m_{1}^{2} & \equiv m_{H_{1}}^{2}+\mu^{2}, \quad m_{2}^{2} \equiv m_{H_{2}}^{2}+\mu^{2}
\end{aligned}
$$


where all parameters correspond to the values at $M_{Z}$.

The condition for the symmetry breaking is given as

$$
m_{1}^{2} m_{2}^{2}<(\mu B)^{2} .
$$

The bounded from below (BFB) condition along the $D$-flat direction requires

$$
m_{1}^{2}+m_{2}^{2}>2|\mu B| .
$$

Further the conditions that minimizing the potential are given as

$$
\begin{aligned}
& m_{1}^{2}+m_{2}^{2}=-\frac{2 \mu B}{\sin 2 \beta}, \\
& m_{1}^{2}-m_{2}^{2}=-\cos 2 \beta\left(M_{Z}^{2}+m_{1}^{2}+m_{2}^{2}\right)
\end{aligned}
$$

where we use the relation $M_{Z}^{2}=\frac{1}{4}\left(g^{2}+g^{\prime 2}\right) v^{2}$.

By the use of stationary conditions (29) and (30), $\mu$ and $B$ are expressed by other parameters $\left(m_{3 / 2}, \cos \theta, \tan \beta, \cdots\right)$, such that

$$
\begin{aligned}
& \frac{|\mu|}{m_{3 / 2}}=\frac{1}{\sqrt{2}}\left(\frac{h-\bar{h}+2 d}{-\cos 2 \beta}-h-\bar{h}-\left(\frac{M_{Z}}{m_{3 / 2}}\right)^{2}\right)^{1 / 2}, \\
& \frac{|B|}{m_{3 / 2}}=\frac{\sin 2 \beta}{2} \frac{m_{3 / 2}}{|\mu|}\left(\frac{h-\bar{h}+2 d}{-\cos 2 \beta}-\left(\frac{M_{Z}}{m_{3 / 2}}\right)^{2}\right) .
\end{aligned}
$$

By the use of RGEs, we can obtain the $\mu$ and $B$ parameters at $M_{X}$ (they are denoted by $\mu_{ \pm}^{(0)}$ and $B_{ \pm}^{(0)}$, respectively.) as follows [20,

$$
\begin{aligned}
& \frac{\mu_{ \pm}^{(0)}}{m_{3 / 2}}= \pm c_{\mu} \frac{|\mu|}{m_{3 / 2}} \\
& c_{\mu} \equiv\left(\frac{\alpha_{2}\left(t_{Z}\right)}{\alpha^{(0)}}\right)^{3 / 2}\left(\frac{\alpha_{1}\left(t_{Z}\right)}{\alpha^{(0)}}\right)^{1 / 22}\left(1+6 \alpha_{t}^{(0)} F\left(t_{Z}\right)\right)^{1 / 4}
\end{aligned}
$$

and

$$
\begin{aligned}
& \frac{B_{ \pm}^{(0)}}{m_{3 / 2}}=\mp \frac{|B|}{m_{3 / 2}}+\frac{\Delta B}{m_{3 / 2}} \\
& \Delta B \equiv 3 A_{t}^{(0)} \frac{\alpha_{t}^{(0)} F\left(t_{Z}\right)}{1+6 \alpha_{t}^{(0)} F\left(t_{Z}\right)} \\
& +M_{1 / 2}^{(0)}\left\{t_{Z}\left(3 \alpha_{2}\left(t_{Z}\right)+\frac{3}{5} \alpha_{1}\left(t_{Z}\right)\right)-\frac{3 \alpha_{t}^{(0)}\left(t_{Z} F^{\prime}\left(t_{Z}\right)-F\left(t_{Z}\right)\right)}{1+6 \alpha_{t}^{(0)} F\left(t_{Z}\right)}\right\}
\end{aligned}
$$


where

$$
\begin{aligned}
& \alpha^{(0)} \equiv \frac{g^{(0) 2}}{4 \pi}, \quad \alpha_{t}^{(0)} \equiv \frac{f_{t}^{(0) 2}}{4 \pi} \\
& F\left(t_{Z}\right) \equiv \int_{0}^{t_{Z}}\left(\frac{\alpha_{3}(t)}{\alpha^{(0)}}\right)^{16 / 9}\left(\frac{\alpha_{2}(t)}{\alpha^{(0)}}\right)^{-3}\left(\frac{\alpha_{1}(t)}{\alpha^{(0)}}\right)^{-13 / 99} d t \\
& t_{Z}=(4 \pi)^{-1} \log \frac{M_{X}^{2}}{M_{Z}^{2}}
\end{aligned}
$$

Here $g^{(0)}$ and $f_{t}^{(0)}$ are the gauge coupling and top Yukawa coupling at $M_{X}$, respectively.

By comparing $B_{ \pm}^{(0)} / m_{3 / 2}$ and $\mu_{ \pm}^{(0)} / m_{3 / 2}$ derived from the stationary conditions of radiative breaking with $B_{p} / m_{3 / 2}$ and $\mu_{p} / m_{3 / 2}(p=Z, \lambda, \mu, M)$ given in the last subsection, we can find allowable parameter regions for $\left(m_{3 / 2}\right.$, $\left.\cos \theta, \Theta_{3}, \Theta_{3}^{\prime}, \tan \beta, d, \lambda, \mu_{\mu}\right)$ leading to successful electroweak symmetry breaking and know which type of $\mu$-term generation mechanism is hopeful. Our strategy, 'bottom-up approach', is more generic and applicable than the usual one, 'top-down approach', where a realization of the radiative breaking scenario is examined by defining the parameters $m_{k}^{2}, \mu$ and $B$ at $M_{X}$ and checking if the quantities renormalized at $M_{Z}$ satisfy stationary conditions (29) $-(30)$.

\section{Which $\mu$-term is hopeful?}

In this section, we examine which type of $\mu$-term generation is hopeful by taking the cases $(\mu-1)-(\mu-3)$ as examples and comparing $B_{ \pm}^{(0)} / m_{3 / 2}$ and $\mu_{ \pm}^{(0)} / m_{3 / 2}$ with $B_{p} / m_{3 / 2}$ and $\mu_{p} / m_{3 / 2}$ determined by $\mu$-term generation mechanism. We also give remarks of several extensions. For our analysis, it is convenient to define the following functions,

$$
\begin{aligned}
& \mathcal{B}_{ \pm p} \equiv B_{ \pm}^{(0)}-B_{p} \\
& \mu_{ \pm p} \equiv \mu_{ \pm}^{(0)}-\mu_{p}
\end{aligned}
$$

The $\mathcal{B}_{ \pm p}$ and $\mu_{ \pm p}$ should be satisfied the conditions $\mathcal{B}_{ \pm p}=0$ and $\mu_{ \pm p}=0$ by definition if we assume the radiative breaking of electroweak symmetry and the $p$-th type of $\mu$-term generation mechanism. 


\subsection{Dilaton dominant case}

First we give allowed regions for $B_{ \pm}^{(0)} / m_{3 / 2}$ and $\mu_{ \pm}^{(0)} / m_{3 / 2}$ in the limit of dilaton dominant SUSY breaking in Fig.1 and 2, respectively. The ranges of $B_{-}^{(0)} / m_{3 / 2}$ and $B_{+}^{(0)} / m_{3 / 2}$ are $-0.74 \sim 0.70$ and $-3.51 \sim-1.45$. The ranges of $\mu_{+}^{(0)} / m_{3 / 2}$ and $\mu_{-}^{(0)} / m_{3 / 2}$ are $3.20 \sim 4.98$ and $-4.98 \sim-3.20$. Here we take $2 \leq \tan \beta \leq 10 \beta, d=0$ and $m_{3 / 2} \geq 50 \mathrm{GeV}$. The value $\tan \beta=2$ (10) corresponds to $B_{-}^{(0)} / m_{3 / 2}=0.70(-0.74), B_{+}^{(0)} / m_{3 / 2}=-3.51(-1.45)$ and $\mu_{ \pm}^{(0)} / m_{3 / 2}= \pm 4.98( \pm 3.20)$. The inequality $m_{3 / 2} \geq 50 \mathrm{GeV}$ is derived from the phenomenological constraint $m_{\tilde{e}} \geq 84 \mathrm{GeV}$ when $n_{e}=-1$. The Fig.3 shows an allowable region of $\left(B_{ \pm}^{(0)} / m_{3 / 2}, d\right)$ in the limit of $m_{3 / 2} \gg M_{Z}$. In this way, we come to a conclusion that the $\mu$-term generation mechanism can be realistic if the values of $B_{p} / m_{3 / 2}$ and $\mu_{p} / m_{3 / 2}$ hit the above ranges.

We study the first case $(\mu-1)$. It is shown that there is no region satisfying $\mathcal{B}_{ \pm Z}=0$ with $d=0$ from Fig. 1 and $B_{Z} / m_{3 / 2}=2$. When $\tan \beta=1.33$, there is a solution of $\mathcal{B}_{ \pm Z}=0$. However, in this case, it is not realistic since the top Yukawa coupling blows up below $M_{X}$. This result is consistent with those in Ref.[14]. Further we find no region satisfying $\mu_{ \pm Z}=0$ with $d=0$ from in Fig.2 and $\mu_{Z} / m_{3 / 2}=1$. As discussed in Ref.[17], $D$-term contribution can survive even in the limit of dilaton dominant SUSY breaking if string model contains an anomalous $U(1)$ symmetry which is cancelled by the GreenSchwarz mechanism [25]. On the other hand, D-term contributions related to anomaly-free symmetries vanish at the tree level in the limit of dilaton dominant SUSY breaking. If we assume the existence of $D$-term contribution, there appears a region consistent with the condition $\mathcal{B}_{ \pm Z}=0$. However it is a very narrow region with a relatively large positive value of $d$. For example, the region with $\tan \beta \sim 2$ and $d \sim 7$ is allowed as given in Fig.3. On the other hand, negative $D$-term contribution is needed to lower the value of $\mu_{+}^{(0)} / m_{3 / 2}$. Hence it is impossible to realize these $\mu$-term generation even with $D$-term contribution.

In the second case $(\mu-2)$, we get the same result for $B$-parameter as the first one. On $\mu$-parameter, we can estimate the value of $\lambda$ using the condition $\mu_{ \pm \lambda}=0$. Hence the radiative breaking scenario can be realized in the models with a relatively large positive $D$-term contribution, i.e., $d=O(10)$.

\footnotetext{
$\S$ In the case that we take a larger $\tan \beta$, it is necessary to incorporate the contribution of Yukawa couplings other than top quark neglected here.
} 
In the third case $(\mu-3)$, we have a solution even in the absence of $D$ term contribution. The allowable region of $\left(m_{3 / 2}, \tan \beta\right)$ is given in Fig.4. The favorable value is $\tan \beta \leq 2.8$ with $d=0$. The introduction of $D$ term contribution yields $\tan \beta>2.8$. The $\mu$-parameter is treated as a free parameter as well as the second one since the origin is unknown.

\subsection{Dilaton and overall moduli case}

We consider the effect of overall moduli $F$-term condensation. We have the same qualitative result for $\cos \theta \neq 0$ as the dilaton dominant case. That is, there is no allowed region in the first case, but very narrow region exists with large positive $d$ in the second case and there exists an allowed region with natural values of $\left(m_{3 / 2}, \tan \beta, d\right)$ in the third case. The above fact can be understood by the use of the Eqs. (31)-(39) directly. That is, both $\mu_{ \pm}^{(0)} / m_{3 / 2}$ and $B_{ \pm}^{(0)} / m_{3 / 2}$ are proportional to $|\sin \theta|$ in the absence of $D$ term contribution and the limit $m_{3 / 2}^{2} \gg M_{Z}^{2}$, and so they decrease as $\cos ^{2} \theta$ increases. Hence it is impossible to satisfy $\mathcal{B}_{ \pm}^{(0)}=0$ in the first and second case with $d=0$. The introduction of $d$ does not improve the situation drastically.

\subsection{Remarks of extension}

We discuss a multi-moduli case (20)-(24). For the case with $\Theta_{3}=1 / \sqrt{3}$ and $\Theta_{3}^{\prime}=0$, we have no allowable regions for $\mathcal{B}_{ \pm}^{(0)}=0$ and $\mu_{ \pm}^{(0)}=0$ because this case corresponds to the first case $(\mu-1)$. We can check that there exists an allowable region with $\tan \beta=2, \Theta_{3}=\Theta_{3}^{\prime}=0.38$ and $\cos ^{2} \theta=0.99$.

We can carry out the case with $n_{H_{1}}=-2$. For $\tan \beta=2$ and $d=0$, we find the following fact. Compared with the case with $n_{H_{1}}=-1$, the value of $B_{-}^{(0)} / m_{3 / 2}$ and $B_{+}^{(0)} / m_{3 / 2}$ decreases and increases, respectively and the absolute value of $\mu_{ \pm}^{(0)} / m_{3 / 2}$ decreases. The difference between the values in the case with $n_{H_{1}}=-1$ and $n_{H_{1}}=-2$ increases as $\cos ^{2} \theta$ increases. Hence the similar conclusion holds for the reality of the radiative breaking scenario as the dilaton dominant case with $n_{H_{1}}=-1$.

In the case of an admixture of several $\mu$-term generation mechanisms, we need a dominant contribution of the third mechanism $(\mu-3)$ to get an allowable region with natural values of $\left(m_{3 / 2}, \cos \theta, \tan \theta, d\right)$. 
We discuss the case $(\mu-4)$. As we have an extra light singlet field $N$, the RG flows of $m_{H_{1}}^{2}$ and $m_{H_{2}}^{2}$ should be modified owing to the effect of the Yukawa coupling $f_{N}$. This case can be applied to a similar strategy discussed in the last section. The difference is that $B_{ \pm}^{(0)}$ receives RGE effects as an $A$ parameter above $M_{Z}$ and so we must compare the renormalized quantity with not $B$-parameter but $A_{N}$ at $M_{X}$. It is not discussed here further because the renormalized quantity contains an unknown parameter $f_{N}$.

\section{Conclusions and Discussions}

We have given a generic method to select a realistic $\mu$-term generation mechanism based on the radiative electroweak symmetry breaking scenario and studied which type is hopeful within the framework of string theory. The $\mu-$ term generated by some non-perturbative effects, i.e., $(\mu-3)$, can be hopeful to realize the radiative symmetry breaking scenario even in dilaton dominant supersymmetry breaking. We have discussed effects of the moduli $F$-term condensation and $D$-term contribution to soft scalar masses. In the case of overall moduli, we have the same qualitative result as in the limit of dilaton dominant SUSY breaking, that is, the first mechanism is impossible to realize the radiative scenario, the second one is required to a large $D$-term contribution of $O\left(10 m_{3 / 2}^{2}\right)$ and the third one is hopeful.

Our method to select a realistic $\mu$-term generation mechanism is so generic and powerful that we can apply it to the case with an improvement of approximation and more complex situations. For example, the improvement by the incorporation of 1-loop effective potential [26], the case with a large $\tan \beta$, the case with large moduli-dominant threshold corrections for gaugino masses, other assignments of modular weight for matter fields and the modular dominant SUSY breaking case. In the above situations, extra contributions $\Delta h$ and $\Delta \bar{h}$ are added to Eqs.(8) and (9), respectively. These studies have to be considered systematically to select a realistic string model.

\footnotetext{
I In [27], $\mu$ and $B$ are studied in the multi-moduli case with a large non-universality between Higgs masses.
} 


\section{Acknowledgments}

The authors are grateful to S. Khalil for useful discussions.

\section{References}

[1] J.E. Kim and H.P. Nilles, Phys. Lett. B138 (1984) 150.

[2] G.F. Giudice and A. Masiero, Phys. Lett. B206 (1988) 480.

[3] H.P. Nilles, M. Srednicki and D. Wyler, Phys. Lett. B120 (1983) 346;

J.M. Frere, D.R.T. Jones and S. Raby, Nucl. Phys. B222 (1983) 11;

J.P. Derendinger and C. Savoy, Nucl. Phys. B237 (1984) 307.

[4] J.E. Kim and H.P. Nilles, Phys. Lett. B263 (1991) 79;

E.J. Chun, J.E. Kim and H.P. Nilles, Nucl. Phys. B370 (1992) 105.

[5] J.A. Casas and C. Muñoz, Phys. Lett. B306 (1993) 288.

[6] See e.g. E. Witten, Nucl. Phys. B433 (1995) 85; Nucl. Phys. B460 (1996) 541;

N. Seiberg and E. Witten, RU-96-12, hep-th/9603003.

[7] L.E. Ibáñez and D. Lüst, Nucl. Phys. B382 (1992) 305.

[8] V.S. Kaplunovsky and J. Louis, Phys. Lett. B306 (1993) 269.

[9] A. Brignole, L.E. Ibáñez and C. Muñoz, Nucl. Phys. B422 (1994) 125.

[10] T. Kobayashi, D. Suematsu, K. Yamada and Y. Yamagishi, Phys. Lett. B348 (1995) 402.

[11] A. Brignole, L.E. Ibáñez, C. Muñoz and C. Scheich, FTUAM 95/26, hep-ph/9508258.

[12] J. Louis and Y. Nir, Nucl. Phys. B447 (1995) 18.

[13] M. Drees, Phys. Lett. B181 (1986) 279;

J.S. Hagelin and S. Kelley, Nucl. Phys. B342 (1990) 95;

A.E. Faraggi, J.S. Hagelin, S. Kelley and D.V. Nanopoulos, Phys. Rev. 
D45 (1992) 3272;

Y. Kawamura, H. Murayama and M. Yamaguchi, Phys. Rev. D51 (1995) 1337 ;

Y. Kawamura, Phys. Rev. D53 (1996) 3779; Prog. Theor. Phys. Supp. 123 (1996) 421.

[14] R. Barbieri, J. Louis and M. Moretti, Phys. Lett. B312 (1993) 451,

A. Brignole, L.E. Ibáñez and C. Muñoz, CERN-TH/96-183, hepph/9607405.

[15] C.Kolda and S.P. Martin, Phys. Rev. D53 (1996) 3871.

[16] A. Font, L.E. Ibáñez, H.P. Nilles and F. Quevedo, Nucl. Phys. B307 (1988) 109;

J.A. Casas, E.K. Katehou and C. Muñoz, Nucl. Phys. B317 (1989) 171;

Y. Kawamura and T. Kobayashi, Nucl. Phys. B481 (1996) 539.

[17] Y. Kawamura and T. Kobayashi, Phys. Lett. B375 (1996) 141; Preprint, DPSU-96-11, INS-Rep-1153, hep-ph/9608233;

Y. Kawamura, T. Kobayashi and T. Komatsu, Preprint, DPSU-96-12, INS-Rep-1161, hep-ph/9609462, to appear in Phys. Lett. B.

[18] J.P. Derendinger, L.E. Ibáñez and H.P. Nilles, Phys. Lett. B155 (1985) 65 ;

M. Dine, R. Rohm, N. Seiberg and E. Witten, Phys. Lett. B156 (1985) 55.

[19] K. Inoue, A. Kakuto, H. Komatsu and S. Takeshita, Prog. Theor. Phys. 68 (1982) 927; 71 (1984) 413;

L.E. Ibáñez, Phys. Lett. B118 (1982) 73; Nucl. Phys. B218 (1983) 514;

L. Alvarez-Gaumé, J. Polchinski and M. Wise, Nucl. Phys. B221 (1983) 495;

J. Ellis, J.S. Hagelin, D.V. Nanopoulos and K. Tamvakis, Phys. Lett. B125 (1983) 275;

L.E. Ibáñez and C. Lopez, Nucl. Phys. B233 (1984) 511;

L.E. Ibáñez, C. Lopez and C. Muñoz, Nucl. Phys. B256 (1985) 218.

[20] K. Hikasa, Minimal Supersymmetry for Collider Physicists, unpublished. 
[21] For review on $\mu$-problem, see, e.g. C. Muñoz, FTUAM 95/20.

[22] L. Dixon, J. Harvey, C. Vafa and E. Witten, Nucl. Phys. B261 (1985) 651; B274 (1986) 285;

L.E. Ibáñez, J.E. Kim, H.P. Nilles and F. Quevedo, Phys. Lett. B191 (1987) 282;

A. Font, L.E. Ibáñez and F. Quevedo, Phys. Lett. B217 (1989) 272;

Y. Katsuki, Y. Kawamura, T. Kobayashi, N. Ohtsubo, Y. Ono and K. Tanioka, Nucl. Phys. B341 (1990) 611;

T. Kobayashi and N. Ohtsubo, Phys. Lett. B262 (1991) 425;

Int. J. Mod. Phys. A9 (1994) 87.

[23] G. Lopes-Cardoso, D. Lüst and T. Mohaupt, Nucl. Phys. B432 (1994) 68 ;

I. Antoniadis, E. Gava, K.S. Narain and T.R. Taylor, Nucl. Phys. B432 (1994) 187.

[24] For review on the supersymmetric Higgs sector, see, e.g. L.E. Ibáñez and G.G. Ross, Perspectives in Higgs Physics, ed. G. Kane.

[25] M.B. Green and J.H. Schwarz, Phys. Lett. B149 (1984) 117;

L.E. Ibáñez, Phys. Lett. B303 (1993) 55.

[26] G. Gamberini, G. Ridolfi and F. Zwirner, Nucl. Phys. B331 (1990) 187.

[27] Y. Kawamura, S. Khalil and T. Kobayashi, Preprint, DPSU-97-4, INSRep-1189, hep-ph/9703239. 


\section{Table Captions}

Table 1 The values of $\alpha_{t}$ and $I_{\Sigma}$. Here we use $m_{t}=175 \mathrm{GeV}$, i.e., $m_{t}\left(m_{t}\right)=$ 167.2GeV and $\alpha_{t}^{(0)}=\alpha_{t}\left(M_{X}\right)$.

Table 2 The formulae of $\mu_{p} / m_{3 / 2}$ and $B_{p} / m_{3 / 2}$. The second column shows the dilaton dominant SUSY breaking case and the third one is the dilaton and overall moduli mixed case. Here we take modular weights $n_{H_{1}}=$ $n_{H_{2}}=-1$.

\section{Table 1}

\begin{tabular}{|c|c|c|c|}
\hline $\tan \beta$ & $\alpha_{t}\left(m_{t}\right)$ & $\alpha_{t}^{(0)}$ & $I_{\Sigma}$ \\
\hline 2 & $9.16 \times 10^{-2}$ & $1.11 \times 10^{-1}$ & 4.179 \\
3 & $8.14 \times 10^{-2}$ & $4.03 \times 10^{-2}$ & 4.140 \\
4 & $7.79 \times 10^{-2}$ & $3.20 \times 10^{-2}$ & 4.104 \\
5 & $7.62 \times 10^{-2}$ & $2.90 \times 10^{-2}$ & 4.083 \\
10 & $7.40 \times 10^{-2}$ & $2.56 \times 10^{-2}$ & 4.051 \\
\hline
\end{tabular}

Table 2

\begin{tabular}{|c|c|c|}
\hline & Dilaton dominant & Dilaton and Moduli \\
\hline$\mu_{Z} / m_{3 / 2}$ & 1 & $1 \pm \cos \theta$ \\
$\mu_{\lambda} / m_{3 / 2}$ & $\lambda$ & $\lambda$ \\
$\mu_{M} / m_{3 / 2}$ & 1 & $1 \pm \sqrt{3} \cos \theta\left(\Theta_{3}+\Theta_{3}^{\prime}\right)$ \\
\hline$B_{Z} / m_{3 / 2}$ & 2 & 2 \\
$B_{\lambda} / m_{3 / 2}$ & 2 & $2 \pm 2 \cos \theta$ \\
$B_{\mu} / m_{3 / 2}$ & $-1 \mp \sqrt{3}$ & $-1-\sqrt{3} \sin \theta \mp \cos \theta$ \\
$B_{M} / m_{3 / 2}$ & 2 & $2 \frac{\left(1 \pm \sqrt{3} \cos \theta \Theta_{3}\right)\left(1 \pm \sqrt{3} \cos \theta \Theta_{3}^{\prime}\right)}{1 \pm \sqrt{3} \cos \theta\left(\Theta_{3}+\Theta_{3}^{\prime}\right)}$ \\
$B_{\mathrm{Mix}} / m_{3 / 2}$ & $\frac{2 m_{3 / 2}(1+\lambda)-\mu_{\mu}(1 \pm \sqrt{3})}{m_{3 / 2}(1+\lambda)+\mu_{\mu}}$ & $\frac{2 m_{3 / 2}(1+\lambda)(1 \pm \cos \theta)-\mu_{\mu}(1+\sqrt{3} \sin \theta \mp \cos \theta)}{m_{3 / 2}(1 \pm \cos \theta+\lambda)+\mu_{\mu}}$ \\
\hline
\end{tabular}




\section{Figure Captions}

Figure 1 The values of $B_{ \pm}^{(0)} / m_{3 / 2}$ versus $m_{3 / 2}$ with $\cos \theta=d=0$.

Figure 2 The values of $\mu_{ \pm}^{(0)} / m_{3 / 2}$ versus $\mu_{3 / 2}$ with $\cos \theta=d=0$.

Figure 3 The values of $B_{ \pm}^{(0)} / m_{3 / 2}$ versus $d$ with $\cos \theta=0$ in the limit of $m_{3 / 2} \gg$ $M_{Z}$.

Figure 4 The values of $\tan \beta$ versus $m_{3 / 2}$ with $\cos \theta=d=0$. 


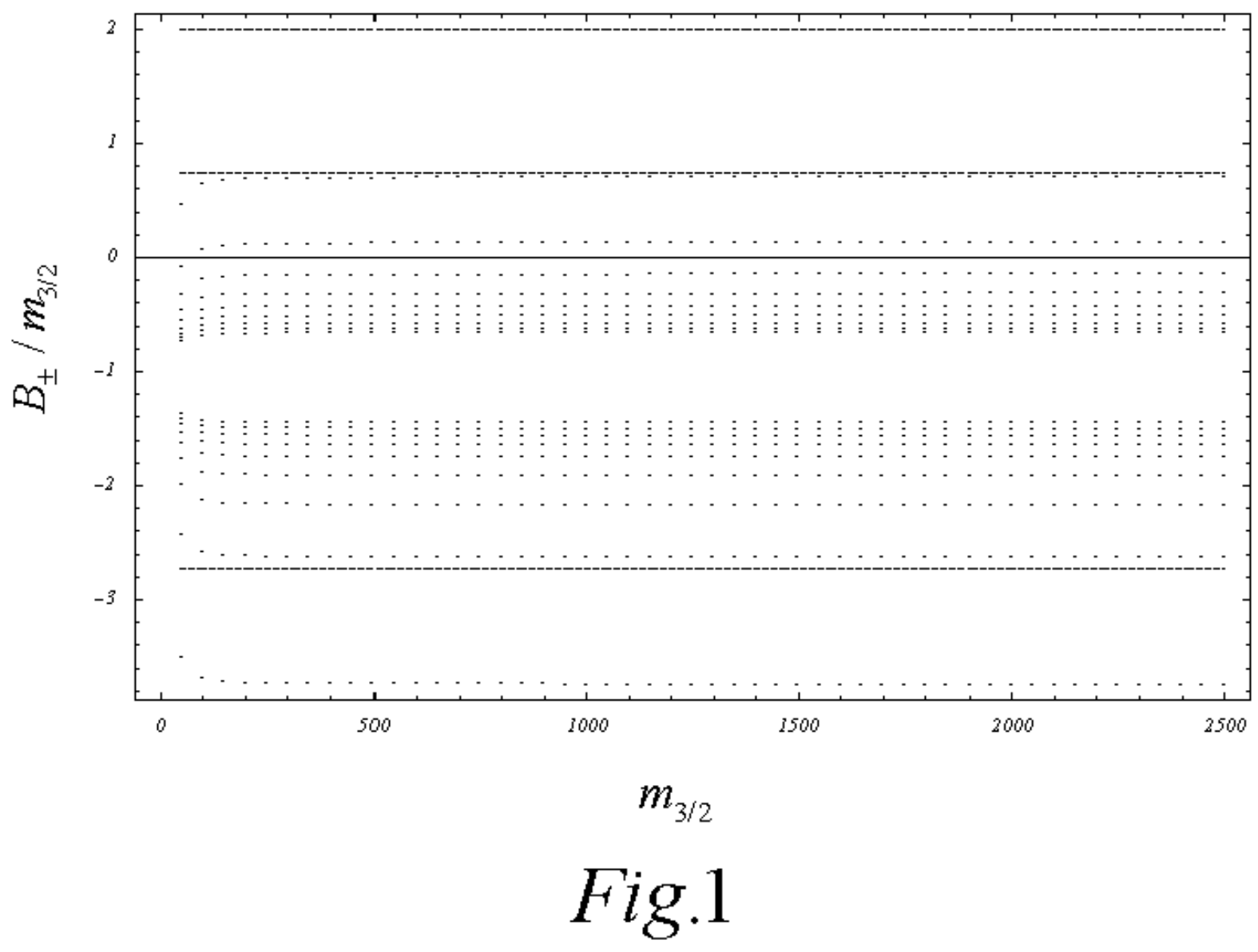




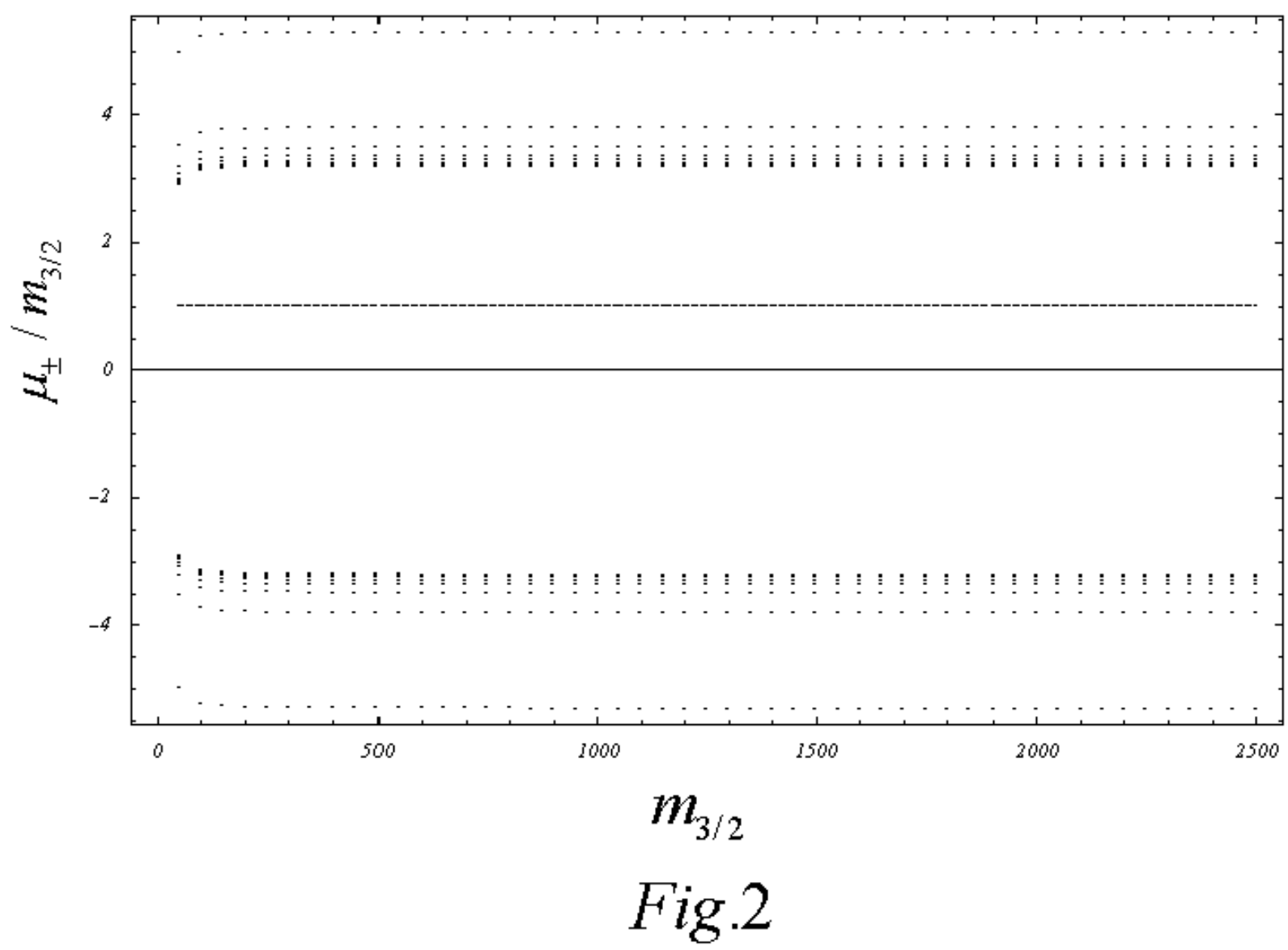




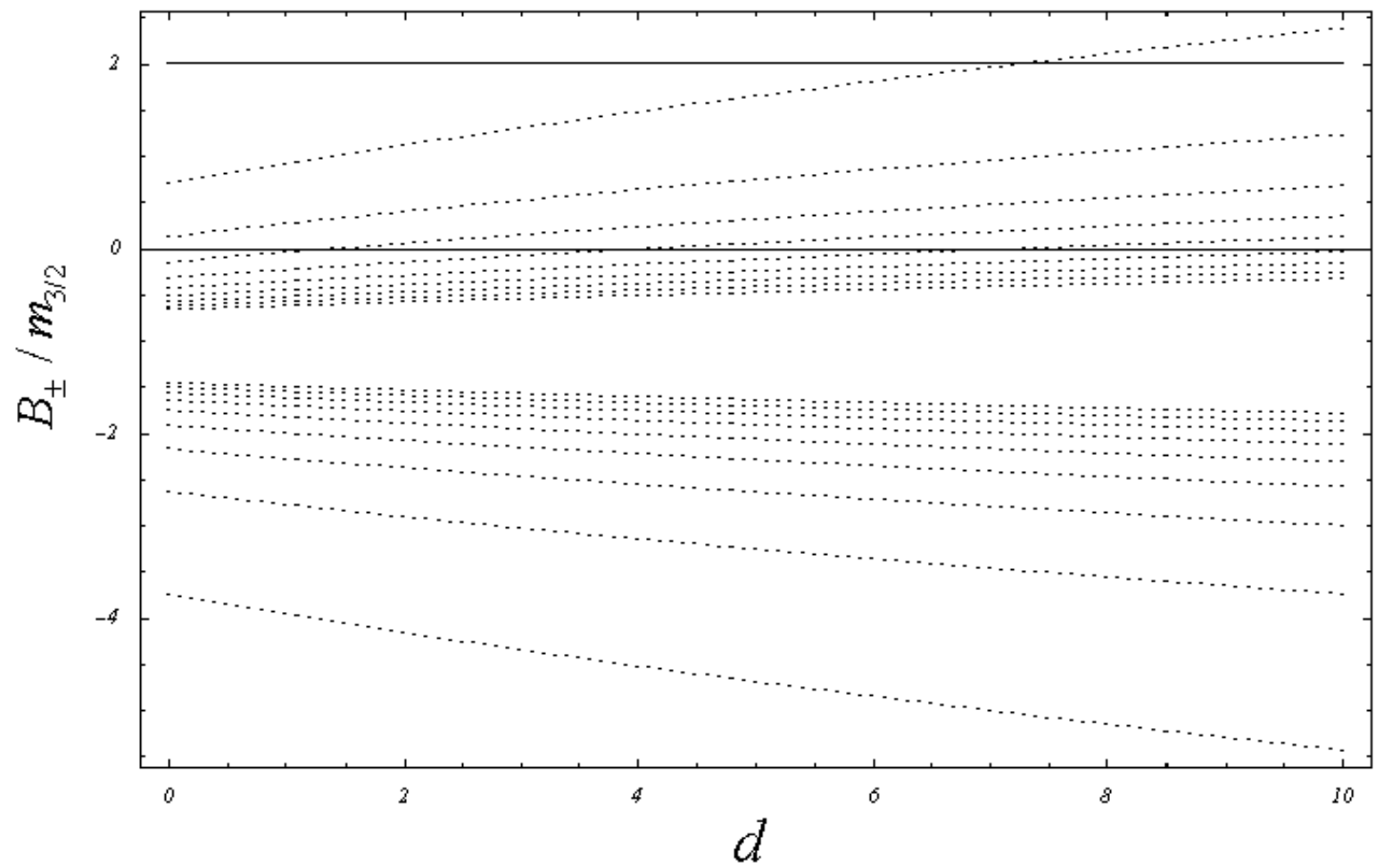

Fig.3 


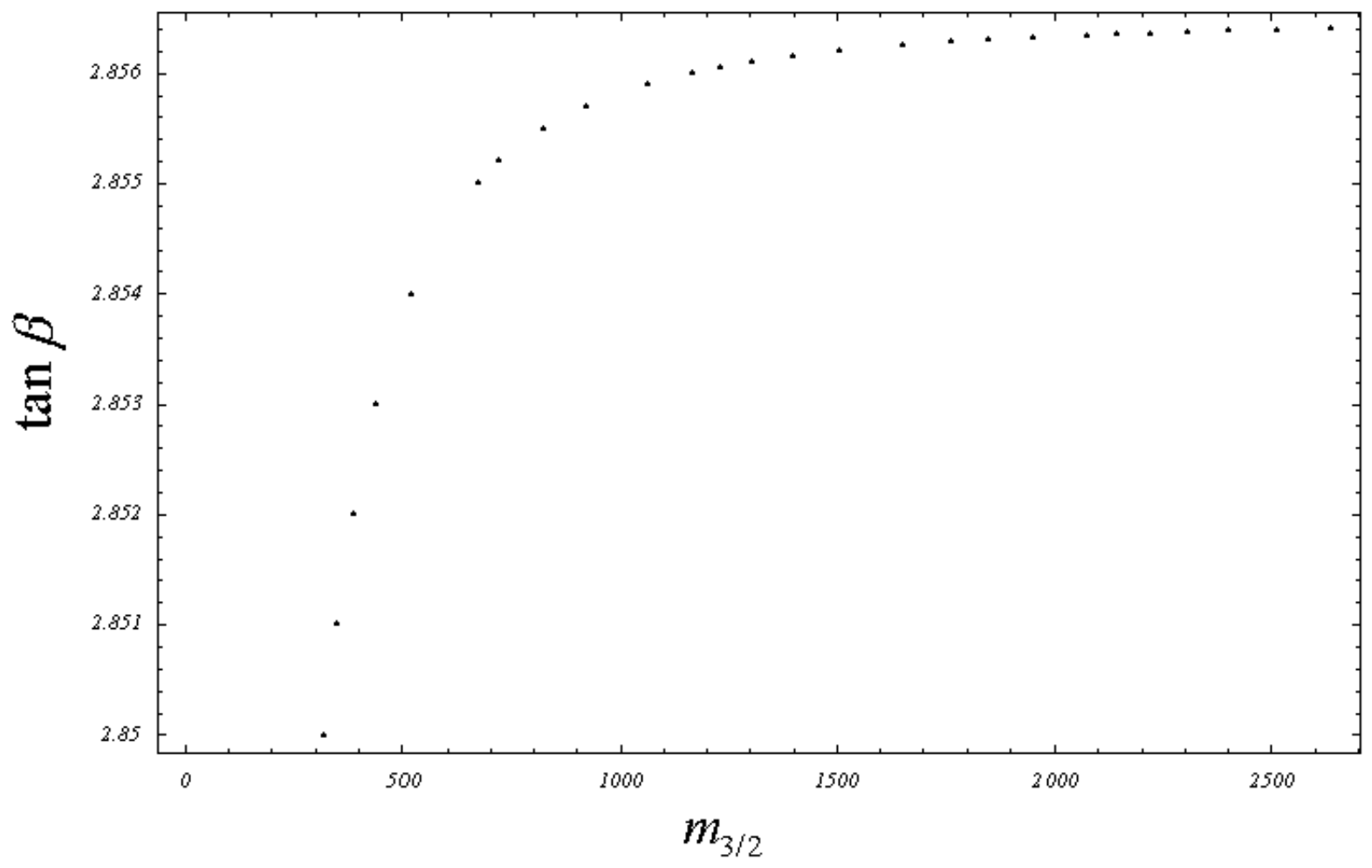

Fig.4 